\title{
Assessment of Fluid Responsiveness in Spontaneously Breathing Patients
}

\author{
J.-L. Teboul, B. Lamia, and X. Monnet
}

\section{Introduction}

Assessment of volume responsiveness is an important issue in patients with spontaneous breathing activity. The difficulty in predicting the response to fluid infusion in this population of patients is variable and depends on the clinical situation. Three different scenarios must be distinguished:

- The first scenario refers to patients admitted to the emergency room for evident acute blood losses or body fluid losses. The diagnosis of hypovolemia is almost certain and the presence of clinical signs of hemodynamic instability (hypotension, tachycardia, oliguria, mottled skin, altered mental status, etc) strongly suggests that a positive hemodynamic response to volume resuscitation will occur, although these signs lack sensitivity. The degree of hypotension, of tachycardia, and of oliguria is important for estimating the degree of hypovolemia and hence the degree of urgency for initiating volume resuscitation.

- The second scenario refers to patients admitted to the emergency room with a high degree of suspicion of septic shock. In this situation, cardiac preload is always inadequate since relative as well as absolute hypovolemia are always present in the early phase of septic shock. The study by Rivers et al. [1] emphasized the importance of volume resuscitation in the first hours of management in this category of patients. There is no need to search for sophisticated parameters to predict volume responsiveness since a positive hemodynamic response is always present at this stage. Rather, there is a need to define parameters that can indicate whether volume infusion should be either continued or stopped because of no further expected efficacy (see the third scenario). There is also a need to define indicators of lung intolerance; however, this is not the subject of the present chapter.

- The third scenario refers to patients hospitalized in the intensive care unit (ICU) who experience hemodynamic instability that requires urgent therapy. In these patients, volume responsiveness is not guaranteed since they have already been volume resuscitated and continuation of volume infusion carries risks of pulmonary edema. In spontaneously breathing patients either without an endotracheal tube or making inspiratory efforts while receiving mechanical ventilation, prediction of volume responsiveness can be a difficult challenge. In these conditions, indices of volume responsiveness that use heart-lung interaction, such as respiratory variations in arterial pressure or in stroke volume and derived indices, are no longer reliable. 


\section{Static Markers of Cardiac Preload as Predictors of Volume Responsiveness}

From the Frank-Starling relationship (ventricular preload vs stroke volume), the response to volume infusion is more likely to occur when the ventricular preload is low than when it is high. Hence, markers of ventricular preload have been suggested for predicting volume responsiveness. This issue has been extensively discussed in a recent review article by Coudray et al. [2]. Therefore, in the present chapter, we will only give a summary of the main parameters.

\section{Cardiac Filling Pressures}

Historically, ventricular filling pressures, namely central venous pressure (CVP) or right atrial pressure (RAP) for the right ventricle, and pulmonary artery occlusion pressure (PAOP) for the left ventricle, have been first proposed as parameters to guide volume resuscitation.

\section{Central venous pressure and right atrial pressure}

These two parameters are assumed to reflect the right ventricular (RV) filling pressure. A few studies have addressed the question of whether CVP or RAP can predict volume responsiveness in critically ill patients $[3,4]$. In most of these studies, a limited number of patients with spontaneous breathing activity were included. In two studies [5, 6], RAP was lower before volume infusion in patients who responded to fluid infusion (in terms of an increase in cardiac output) than in non-responders. However, a small percentage of patients were studied while breathing spontaneously ( $6 \%$ and $33 \%$, respectively). In addition, the study by Wagner and Leatherman [6], reported a weak correlation between pre-infusion RAP and changes in stroke volume induced by fluid infusion. For example, a fluid-induced increase in stroke volume by $25 \%$ was observed for a pre-infusion RAP value of $2 \mathrm{mmHg}$ as well as for a value of $13 \mathrm{mmHg}$ [6]. Conversely, a RAP value of $11 \mathrm{mmHg}$ was associated with a fluid-induced increase in stroke volume ranging from 10 to $45 \%$ [6]. In other studies including patients with spontaneous breathing activity, there was no relationship between the initial RAP and the response to volume infusion [7-11]. All these studies, therefore, suggest that RAP is a poor predictor of volume responsiveness for the general population of critically ill patients including those who exhibit spontaneous breathing activity, although the total number of patients included in the available studies is quite low.

\section{Pulmonary artery occlusion pressure}

The PAOP is the pressure obtained after inflating the distal balloon of a pulmonary artery catheter in a large branch of the pulmonary artery. Since a static column is created between the inflated balloon and the venous site where the blood flow resumes, PAOP is assumed to reflect the pressure in a large pulmonary vein and, thus, the left atrial pressure and eventually the left ventricular (LV) end diastolic pressure (LVEDP) [12]. Obtaining a meaningful measurement can be a difficult challenge in dyspneic patients who experience large swings in intrathoracic pressure. In order to limit the influence of the intrathoracic pressure on PAOP measurements, it is recommended that PAOP be measured at the end-expiratory period. However, in patients receiving mechanical ventilation who exhibit inspiratory efforts, the endexpiratory period is not always easy to identify [13]. 
Provided that reliable measurements are obtained, PAOP is considered as a measure of LV preload, although preload is assumed to fit better with end-diastolic volume than with end-diastolic pressure of the ventricle [12]. A few studies have addressed the question of whether PAOP can predict volume responsiveness in critically ill patients [3, 4]. Six studies, in which $16 \%$ [14], 24\% [8], 33\% [5], 36\% [9], $54 \%$ [7], and $57 \%$ [10] of the patients were breathing spontaneously, showed no relationship between the pre-infusion PAOP and the hemodynamic response to fluid. In one study, in which just $6 \%$ the patients were breathing spontaneously, the pre-infusion PAOP value was lower in responders but a weak correlation was found between PAOP and the increase in stroke volume induced by volume infusion $\left(r^{2}=0.33\right)$; no cut-off value was found to discriminate responders from non-responders [6]. Finally, in one study, the pre-infusion PAOP value was surprisingly higher in the patients who subsequently responded to fluid infusion [15]. No clear explanation was given by the authors of this study, which included only four patients who were breathing spontaneously [15].

In summary, there is no evidence that the pre-infusion PAOP can predict volume responsiveness in critically ill patients, although the total number of patients with spontaneous breathing activity included in the available studies is less than 80 .

\section{End-diastolic Ventricular Dimensions}

Since ventricular end-diastolic dimensions are considered as better indicators of cardiac preload than filling pressures, measurements of ventricular dimensions have also been proposed for predicting fluid responsiveness.

\section{Right ventricular end-diastolic volume}

The RV end-diastolic volume (RVEDV) can be evaluated at the bedside by fast response pulmonary artery catheters. In two studies, in which $16 \%$ [14] and $31 \%$ [15] of patients were breathing spontaneously, Diebel et al. reported lower values of RVEDV index (RVEDVI) in responder than in non-responder patients and suggested that a beneficial hemodynamic effect of volume expansion was likely when the RVEDVI was below $90 \mathrm{ml} / \mathrm{m}^{2}$ and very unlikely when the RVEDVI was greater than $138 \mathrm{ml} / \mathrm{m}^{2}$. However, when the RVEDVI ranged between 90 and $138 \mathrm{ml} / \mathrm{m}^{2}$, which is probably the most frequent occurrence in critically ill patients who are already resuscitated, no cut-off value could be proposed to discriminate responder and nonresponder patients $[14,15]$. In two other studies, in which $6 \%$ and $24 \%$ of patients were breathing spontaneously, no significant difference was observed between responders and non-responders with respect to the baseline value of the RVEDVI $[6,8]$.

In two older studies, including 54\% [7] and 33\% [5] of patients breathing spontaneously, RVEDV was calculated after assessing the RV ejection fraction (RVEF) using cardiac scintigraphy and after measuring cardiac output using thermodilution. In these studies, no significant difference was observed between responders and non-responders with respect to the pre-infusion value of RVEDV [5, 7].

\section{Left ventricular end-diastolic dimensions}

Cardiac scintigraphy and echocardiography are used to estimate LV end-diastolic volume (LVEDV) and LV end-diastolic area (LVEDA). Two old studies including patients with spontaneous breathing indicated that LVEDV was of poor value for predicting volume responsiveness $[5,7]$. To our knowledge, no study has examined 
the significance of LVEDV or LVEDA obtained with echocardiography in patients who breathe spontaneously. It should be remembered that in deeply sedated patients receiving mechanical ventilation, the LVEDA was reported to be unreliable for assessing volume responsiveness [16-19].

\section{Global end-diastolic volume}

The global end-diastolic volume (GEDV) is obtained by the transpulmonary thermodilution method (PiCCO monitoring system). Regarding the prediction of volume responsiveness, no study using this parameter has been performed in patients breathing spontaneously. It must be remembered that in patients receiving mechanical ventilation, the GEDV was lower in volume responders than in non-responders and that the lower the pre-infusion GEDV, the more likely the positive hemodynamic response [20]. However, GEDV values in responders and non-responders overlapped and no cut-off value could identify responders and non-responders with high sensitivity and specificity values [20].

\section{Why do Static Markers of Preload Fail to Predict Volume Responsiveness?}

\section{Markers of preload are not always accurate measures of cardiac preload}

This is particularly the case in the following situations:

- In the presence of mitral stenosis or mitral insufficiency, PAOP can be higher than the LVEDP. The value measured just before the ' $v$ ' wave upslope must be taken as an estimate of LVEDP in the case of mitral insufficiency.

- In the presence of intrinsic positive end-expiratory pressure (PEEP), PAOP differs from LV filling pressure, even when the measurement is made during the end-expiratory period [21].

- Even when PAOP reflects the LV filling pressure, it can still be a poor marker of LV preload in the case of reduced LV compliance where achieving high filling pressures can be necessary to maintain optimal levels of LV preload [22].

- In the presence of tricuspid regurgitation, thermodilution RVEDV measurements can be erroneous [23].

\section{Assessment of preload is not assessment of preload responsiveness}

More generally, there is a physiological reason explaining why even the most accurate static marker of preload will never be a reliable predictor of preload-responsiveness. Indeed, the slope of the Frank-Starling curve depends on the systolic cardiac function. In this regard, a given value of preload can be associated with preloaddependence and hence with volume responsiveness in normal conditions (steep part of the Frank-Starling curve) or with preload-independence and hence with absence of volume responsiveness in the case of decreased cardiac contractility (flat part of the Frank-Starling curve).

\section{Summary}

Static markers of preload like CVP, RAP, PAOP, RVEDV, LVEDV, and GEDV are not accurate predictors of volume responsiveness in spontaneously breathing patients as they are in patients receiving mechanical ventilation without exhibiting inspiratory efforts [3, 4, 24]. Importantly, even if an accurate static measure of preload were available, it would not be possible to use it to reliably predict volume responsiveness. However, the following important points should be stressed: First, the available clini- 
cal studies that have addressed the issue of volume responsiveness are scarce and did not include a lot of patients with spontaneous breathing activity. In addition, the specific behavior of those patients was not distinguished from the behavior of patients without spontaneous breathing activity. Therefore, the results of these studies must be interpreted with caution. Second, in the available studies, patient selection for fluid challenge and the method used to perform the fluid challenges (volume and type of fluid, duration of the trial, definition of fluid response) were quite heterogeneous. Third, in addition, in these studies, almost all the patients were studied after they had already been resuscitated, such that the values of preload markers were rarely low before the volume challenge was done. Thus, one cannot exclude that low values of CVP and/or PAOP and/or other markers of cardiac preload, potentially measured in non-resuscitated shocked patients, may still be associated with a positive hemodynamic response to volume loading. For example, in the study by Michard et al. [20] (in patients receiving mechanical ventilation), the rate of positive response to fluid was markedly higher $(77 \%)$ in the subgroup of patients with low GEDV index values $\left(<610 \mathrm{ml} / \mathrm{m}^{2}\right)$ compared to the subgroup of patients with intermediate GEDV index values (between 610 and $815 \mathrm{ml} / \mathrm{m}^{2}$ ) and the subgroup of patients with high GEDV index values $\left(>815 \mathrm{ml} / \mathrm{m}^{2}\right.$ ) (rates of response: $43 \%$ and $23 \%$, respectively). Interestingly, the four patients with a GEDV index $<500 \mathrm{ml} / \mathrm{m}^{2}$ responded to volume loading while all five patients with a GEDV index $>950 \mathrm{ml} / \mathrm{m}^{2}$ did not respond to volume loading [20]. Similar findings with RAP as a static measure of preload have been recently reported by Heenen et al. [10]. On the other hand, in the available studies, volume challenges were rarely done in patients with high values of CVP and/or PAOP at baseline, mainly for ethical reasons. Thus, one cannot exclude that high values of markers of preload are associated with the absence of a hemodynamic response to volume loading. For example, in the study by Michard et al. [20], the percentage of positive response to fluid was very low in the subgroup of patients with high GEDV index values $\left(>850 \mathrm{ml} / \mathrm{m}^{2}\right)$. Similarly, in the study by Heenen et al., high values of pre-infusion RAP were associated with a low rate of positive response to volume administration [10]. Although the markers of preload do not appear to be good predictors of volume responsiveness, most available studies have shown that these markers did increase after volume challenge in responders as well as in non-responders. This finding suggests that these static indexes are valuable tools to confirm that the volume infused actually reaches the cardiac chambers, and, therefore, that these indexes do inform about changes in cardiac preload [2].

\section{Dynamic Parameters}

\section{Arterial Pressure and Stroke Volume Respiratory Variation}

Numerous studies have demonstrated that dynamic indexes, such as respiratory variation in arterial pressure and in stroke volume, are valuable for predicting volume responsiveness in patients receiving mechanical ventilation, provided that they do not experience any active breathing efforts and/or cardiac arrhythmias and that they do not receive too low a tidal volume $[3,4]$. Therefore, the findings of these studies cannot be extrapolated to patients receiving mechanical ventilation while exhibiting inspiratory efforts or to patients who breathe spontaneously without any mechanical support. To our knowledge, only three studies have investigated whether or not arterial pulse pressure variation (PPV) was predictive of volume responsiveness in such 
groups of patients. In a study by Monnet et al, a PPV $\geq 12 \%$ predicted volume responsiveness with a sensitivity of $88 \%$ and a specificity of $93 \%$ in the subgroup of deeply sedated patients receiving mechanical ventilation [25]. By contrast, PPV had no predictive value in the subgroup of patients with ventilator triggering [25]. This latter important conclusion has been recently confirmed in the study by Heenen et al. [10]. In a study that included non-intubated patients, a PPV value $>12 \%$ was shown to reliably predict a beneficial response to volume infusion in the case of quiet spontaneous breathing [11]. However, that study also showed that PPV was not valuable for predicting volume responsiveness when its baseline value was less than $12 \%$ and/or when patients were dyspneic [11].

\section{Inspiratory Decrease in Right Atrial Pressure}

During a normal spontaneous inspiration, the intrathoracic pressure decreases and this will eventually result in a decrease in RAP relative to the extrathoracic vessel compartment and, thus, in an increased venous return to the left atrium and an increased RV stroke volume if the right ventricle is preload-dependent. As a result, an increase in LV stroke volume will be expected with a time delay of a few heart beats, provided that the left ventricle is also preload-dependent. Magder et al. $[9,26]$ hypothesized that when the right ventricle is preload-independent, not only will the right and subsequently the left stroke volume not increase with volume loading, but the RAP will also not decrease during normal inspiration. In their first study, the authors included a heterogeneous population of patients: $36 \%$ of them experienced total spontaneous breathing and $64 \%$ of them received mechanical ventilation but were able to breathe spontaneously after disconnection from the ventilator [9]. The decrease in RAP was measured during a spontaneous inspiration (after a short disconnection from the ventilator in those receiving mechanical ventilation). An inspiratory decrease in RAP ( $\triangle \mathrm{RAP})$ of at least $1 \mathrm{mmHg}$ was predictive of a positive response to volume loading with positive predictive values of $77 \%$ [26] and $84 \%$ [9] and negative values of $81 \%$ [26] and $93 \%$ [9].

To our knowledge, this index is not widely used in clinical practice. One of the major problems that limits its use is that the patient must generate a sufficiently deep decrease in intrathoracic pressure for a correct interpretation. In the two studies by Magder et al., this was ensured by a decrease in PAOP by at least $2 \mathrm{mmHg}$ during inspiration after postulating that the decrease in PAOP reliably reflects the decrease in intrathoracic pressure $[9,26]$. Therefore, the use of $\triangle R A P$ requires the insertion of a pulmonary artery catheter, which obviously represents a true limitation.

Contrary to the results of Magder et al. $[9,26]$, Heenen et al. reported that $\triangle$ RAP was not predictive of volume responsiveness in patients with spontaneous breathing activity with or without mechanical support [10]. However, this issue is still a matter of debate [27].

\section{Passive Leg Raising}

\section{Description and Interpretation}

Passive leg raising is a maneuver that transiently and reversibly increases venous return by shifting venous blood from the legs to the intrathoracic compartment [28, 29]. Passive leg raising ( $45^{\circ}$ elevation) results in an increase in right [30] and left 
[31] ventricular preload. Passive leg raising can, therefore, mimic the effects of fluid loading and has been proposed for a long time as a first line therapy of hypovolemic shock ('autotransfusion' effect). The way in which passive leg raising alters preload is probably by an increase in the mean systemic pressure, the driving force for venous return, due to the gravitational shift of venous blood from unstressed to stressed volume. This mechanism is probably of major importance during passive leg raising in patients receiving mechanical ventilation because the volume of blood enclosed by the thoracic and splanchnic beds is already stressed by positive airway pressure and these vascular compartments are less compliant than when mechanical ventilation is not used [32]. In these conditions, the increase in mean systemic pressure with passive leg raising is expected to be higher in mechanically ventilated patients than in non-mechanically ventilated patients. However, the effects of passive leg raising on cardiac output are variable [30, 33-35], probably depending on the degree of leg elevation and on the existence of cardiac preload reserve. In this regard, Boulain et al. [36] reported, in deeply sedated patients receiving mechanical ventilation, that the increase in stroke volume induced by passive leg raising occurred only in patients who increased their stroke volume in response to a subsequent $300 \mathrm{ml}$ volume infusion. In patients who did not respond to volume loading, passive leg raising did not change stroke volume despite increases in RAP and PAOP. Thus, passive leg raising, which was able to increase cardiac preload in all the studied patients, increased stroke volume only in those with cardiac preload-dependence. Hence, passive leg raising can be proposed as a test to detect fluid responsiveness in critically patients. It is interesting to note that in the above-mentioned clinical study, the changes in PAOP induced by passive leg raising were immediately and fully reversible when the patients' legs were laid down [36]. This finding suggests that passive leg raising may help in predicting individual fluid responsiveness while avoiding the hazards of unnecessary fluid loading. Since it does not require any analysis of respiratory changes in stroke volume or its surrogates, passive leg raising is potentially usable in patients experiencing spontaneous breathing activity or arrhythmias.

\section{Clinical Use}

\section{Changes in arterial pulse pressure}

Theoretically, the best marker of the hemodynamic response to passive leg raising as a predictor of the hemodynamic response to fluid loading would be a significant increase in stroke volume. Because arterial pulse pressure is directly proportional to LV stroke volume and assuming that arterial compliance is not altered by passive leg raising, an increase in pulse pressure during passive leg raising should indicate an increase in stroke volume and thus a positive response to fluid infusion. However, in a study by Boulain et al. [36], the correlation between passive leg raising-induced changes in radial pulse pressure and fluid loading-induced changes in stroke volume was only fair. This observation was likely because changes in radial pulse pressure may not reflect changes in aortic pulse pressure owing to the potential occurrence of complex changes in pressure wave propagation and reflection during change in blood flow induced by passive leg raising. In addition, a given change in stroke volume in different patients with different aortic compliance may be reflected by different changes in aortic pulse pressure. 


\section{Changes in 'pulse contour' cardiac output}

A more direct measurement of stroke volume during passive leg raising should be more relevant to detect volume responsiveness. Because of the short period of this test (less than 1 minute), the thermodilution method is not appropriate even in its automatic and semi-continuous mode. Indeed, this method takes at least 10 minutes to completely detect a given change in cardiac output [37]. A beat-to-beat basis for measuring stroke volume should be a better approach for tracking rapid changes of stroke volume induced by passive leg raising. Technologies using pulse contour analysis - like the PiCCO system - would be appropriate for that purpose. To our knowledge, no study has yet been published on the effects of passive leg raising on PiCCO stroke volume for the prediction of fluid responsiveness. In a preliminary study performed in patients with spontaneous breathing activity and/or arrhythmias, the increase in pulse contour cardiac index induced by passive leg raising correlated nicely with the increase in pulse contour cardiac index induced by subsequent volume loading $(\mathrm{r}=0.62, \mathrm{p}<0.05)$ while the passive leg raising-induced increase in pulse pressure correlated only weakly with the fluid-induced increase in pulse pressure $(\mathrm{r}=0.40 \mathrm{p}=0.03)$ [38]. An increase in cardiac index $>12 \%$ during passive leg raising predicted fluid responsiveness (defined by a fluid-induced increase in cardiac index $\geq 15 \%$ ) with a sensitivity of $70 \%$ and a specificity of $92 \%$ (Fig. 1), values significantly better than those reported for the prediction of volume responsiveness by passive leg raising-induced increase in pulse pressure (50\% and $80 \%$, respectively) [38].

\section{Changes in aortic blood flow}

An alternative beat-to-beat based monitoring method is the esophageal Doppler. Recent technologic developments allow continuous measurement of both the descending aortic blood velocity (Doppler method) and the diameter of the descending aorta (time-motion echographic transducer). Therefore, it is now possible to monitor blood flow in the descending aorta.

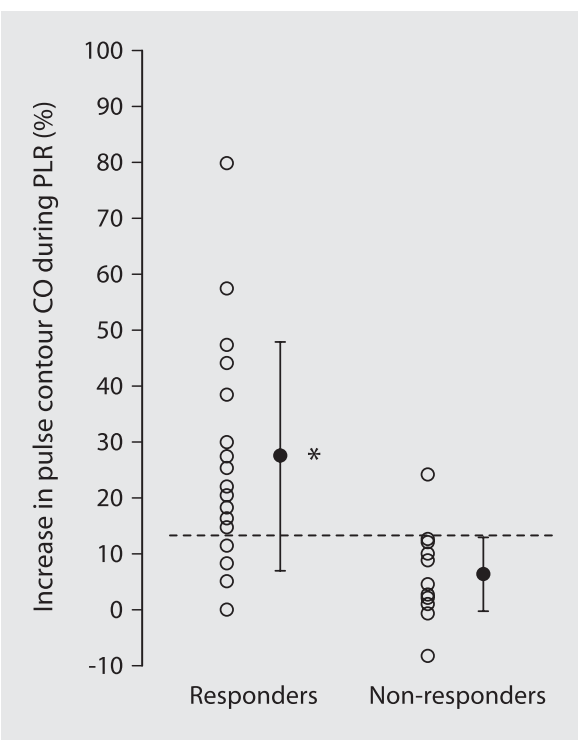

Fig. 1. Representation of individual values of percent changes in pulse contour cardiac output (CO) in response to passive leg raising (PLR) in responders to volume challenge and in nonresponders. Responders to volume challenge were defined as patients who increased their pulse contour cardiac output by more than $15 \%$ after receiving fluid infusion. An increase in pulse contour cardiac output by $12 \%$ during passive legraising was the best cut-off value for discriminating non-responders and responders to volume challenge (see text). 
In a study performed in 71 mechanically ventilated patients considered for volume expansion, we hypothesized that the increase in descending aortic blood flow in response to passive leg raising would predict fluid responsiveness [25]. Thirty-one patients had spontaneous breathing activity and/or arrhythmias. In 37 patients (responders), the aortic blood flow increased by $\geq 15 \%$ after fluid infusion. A passive leg raising-induced increase in the aortic blood flow $\geq 10 \%$ predicted fluid responsiveness with a sensitivity of $97 \%$ and a specificity of $94 \%$ (area under the receiver operating characteristic [ROC] curve: $0.96 \pm 0.02$ ). These excellent predictive values were quite similar in the group of patients with spontaneous breathing activity (area under the ROC curve: $1.00 \pm 0.00$ ) and in the group of deeply sedated patients (area under the ROC curve: $0.91 \pm 0.06$ ) [25]. Interestingly, a passive leg raising-induced increase in pulse pressure $\geq 12 \%$ predicted volume responsiveness with significantly lower sensitivity $(60 \%)$ and specificity $(85 \%)$ (area under the ROC curve: $0.75 \pm 0.06$ ) [25]. This only fair prediction was similar in the group of spontaneously breathing patients (area under the ROC curve: $0.69 \pm 0.13$ ) and in the group of deeply sedated patients (area under the ROC curve: $0.74 \pm 0.09$ ) [25]. This study suggests that measuring changes in aortic blood flow rather than pulse pressure during passive leg raising is a more robust indicator of preload-responsiveness in a general population of mechanically ventilated patients including those with spontaneous inspiratory efforts and/or arrhythmias.

\section{Conclusion}

In spontaneously breathing patients (with or without mechanical ventilation), the prediction of volume responsiveness can be a difficult challenge, in particular in those who have already been resuscitated in the preceding hours or days and in whom continuation of fluid infusion carries risks of pulmonary edema. In these cases, static markers of cardiac preload are generally in the normal range and are rarely helpful for determination of volume responsiveness. Since absolute measures of preload cannot be used effectively to assess volume responsiveness, more dynamic tests need to be employed to improve the utility of these measures [39]. Because of the presence of spontaneous breathing, the indices of volume responsiveness that use heart-lung interactions, such as respiratory variation in arterial pressure and in stroke volume are no longer reliable. Careful analysis of the hemodynamic consequences of passive leg raising using real-time aortic blood flow monitoring may be helpful for predicting the beneficial effects of volume administration. In the most difficult cases, a fluid challenge strategy can still be applied provided that clinicians carefully follow the recommended rules in terms of the type of fluid, rate of infusion, clinical end-points, and safety limits in order to minimize the risks of fluid overload [40].

\section{References}

1. Rivers E, Nguyen B, Havstad S, et al (2001) Early goal-directed therapy in the treatment of severe sepsis and septic shock. N Engl J Med 345:1368-1377

2. Coudray A, Romand JA, Treggiari M, Bendjelid K (2005) Fluid responsiveness in spontaneously breathing patients: A review of indexes used in intensive care. Crit Care Med 33:2757-2762

3. Michard F, Teboul JL (2002) Predicting fluid responsiveness in ICU patients. A critical analysis of the evidence. Chest 121: 2000-2008 
4. Bendjelid K, Romand JA (2003) Fluid responsiveness in mechanically ventilated patients: a review of indices used in intensive care. Intensive Care Med 29:352-60

5. Schneider AJ, Teule GJJ, Groeneveld ABJ, Nauta J, Heidendal GA, Thijs LG (1988) Biventricular performance during volume loading in patients with early septic shock, with emphasis on the right ventricle: a combined hemodynamic and radionuclide study. Am Heart J 116: $103-112$

6. Wagner JG, Leatherman JW (1998) Right ventricular end-diastolic volume as a predictor of the hemodynamic response to a fluid challenge. Chest 113:1048-1054

7. Calvin JE, Driedger AA, Sibbald WJ (1981) The hemodynamic effect of rapid fluid infusion in critically ill patients. Surgery 90:61-76

8. Reuse C, Vincent JL, Pinsky MR (1990) Measurements of right ventricular volumes during fluid challenge. Chest 98:1450-1454

9. Magder S, Georgiadis G, Cheong T (1992) Respiratory variations in right atrial pressure predict the response to fluid challenge. J Crit Care 7:76-85

10. Heenen S, De Backer D, Vincent JL (2006) How can the response to volume expansion in patients with spontaneous respiratory movements be predicted? Crit Care 10:R102

11. Soubrier S, Saulnier F, Hubert H, et al (2007) Usefulness of dynamic indicators to predict fluid responsiveness in spontaneously breathing critically ill patients. Intensive Care Med (in press)

12. Monnet X, Teboul JL (2006) Invasive measures of preload. Curr Opin Crit Care 12:235-240

13. Rizvi K, Deboisblanc BP, Truwit JD, et al (2005) Effect of airway pressure display on interobserver agreement in the assessment of vascular pressures in patients with acute lung injury and acute respiratory distress syndrome. Crit Care Med 33:98-103

14. Diebel L, Wilson RF, Heins J, Larky H, Warsow K, Wilson S (1994) End-diastolic volume versus pulmonary artery wedge pressure in evaluating cardiac preload in trauma patients. J Trauma 37:950-955

15. Diebel LN, Wilson RF, Tagett MG, Kline RA (1992) End-diastolic volume. A better indicator of preload in the critically ill. Arch Surg 127:817-822

16. Tavernier B, Makhotine O, Lebuffe G, Dupont J, Scherpereel P (1998) Systolic pressure variation as a guide to fluid therapy in patients with sepsis-induced hypotension. Anesthesiology $89: 1313-1321$

17. Tousignant CP, Walsh F, Mazer CD. The use of transesophageal echocardiography for preload assessment in critically ill patients. Anesth Analg 2000; 90:351-355

18. Feissel M, Michard F, Mangin I, Ruyer O, Faller JP, Teboul JL (2001) Respiratory changes in aortic blood velocity as an indicator of fluid responsiveness in ventilated patients with septic shock. Chest 119:867-873

19. Preisman S, Kogan S, Berkenstadt H, Perel A (2005) Predicting fluid responsiveness in patients undergoing cardiac surgery: functional haemodynamic parameters including the Respiratory Systolic Variation Test and static preload indicators. Br J Anaesth 95:746-755

20. Michard F, Alaya S, Zarka V, Bahloul M, Richard C, Teboul JL (2003) Global end-diastolic volume as an indicator of cardiac preload in patients with septic shock. Chest 124:1900-1908

21. Teboul JL, Pinsky MR, Mercat A, et al (2000) Estimating cardiac filling pressure in mechanically ventilated patients with hyperinflation. Crit Care Med 28:3631-3636

22. Crexells C, Chatterjee K, Forrester JS, Dikshit K, Swan HJ (1973) Optimal level of filling pressure in the left side of the heart in acute myocardial infarction. N Engl J Med 289:1263- 1266

23. Cigarroa RG, Lange RA, Williams RH, Bedotto JB, Hillis LD (1989) Underestimation of cardiac output by thermodilution in patients with tricuspid regurgitation. Am J Med $86: 417-420$

24. Osman D, Ridel C, Ray P, et al (2006) Cardiac filling pressures are not appropriate to predict hemodynamic response to volume challenge. Crit Care Med 35:64-68

25. Monnet X, Rienzo M, Osman D, et al (2006) Passive leg raising predicts fluid responsiveness in the critically ill. Crit Care Med 34:1402-1407

26. Magder S, Lagonidis D (1999) Effectiveness of albumin versus normal saline as a test of volume responsiveness in post-cardiac surgery patients. J Crit Care 14:164-171

27. Magder S (2006) Predicting volume responsiveness in spontaneously breathing patients: still a challenging problem. Crit Care 10:165

28. Rutlen DL, Wackers FJT, Zaret BL (1981) Radionuclide assessment of peripheral intravascular 
capacity: a technique to measure intravascular volumes changes in the capacitance circulation in man. Circulation 64:146-152

29. Reich DL, Konstadt SN, Raissi S, Hubbard M, Thys DM (1989) Trendelenburg position and passive leg raising do not significantly improve cardiopulmonary performance in the anesthetized patient with coronary artery disease. Crit Care Med 17:313-317

30. Thomas M, Shillingford J (1965) The circulatory response to a standard postural change in ischaemic heart disease. Br Heart J 27:17-27

31. Rocha P, Lemaigre D, Leroy M, De Zutterre D, Liot F (1987) Nitroglycerin-induced decrease of carbon monoxide diffusion capacity in acute myocardial infarction reversed by elevating legs. Crit Care Med 15:131-133

32. Chihara E, Hashimoto S, Kinoshita T, Hirose M, Tanaka Y, Morimoto T (1992) Elevated mean systemic filling pressure due to intermittent positive-pressure ventilation. Am J Physiol 262:H1116-H1121

33. Wong DH, Tremper KK, Zaccari J, Hajduczek J, Konchigeri HN, Hufstedler SM (1988) Acute cardiovascular response to passive leg raising. Crit Care Med 16:123-125

34. Wong DH, O'Connor D, Tremper KK, Zaccari J, Thompson P, Hill D (1989) Changes in cardiac output after acute blood loss and position change in man. Crit Care Med 17:979-983

35. Gaffney FA, Bastian BC, Thal ER, Atkins JM, Blomqvist CG (1982) Passive leg raising does not produce a significant or sustained autotransfusion effect. J Trauma 22:190-193

36. Boulain T, Achard JM, Teboul JL, Richard C, Perrotin D, Ginies G (2002) Changes in blood pressure induced by passive leg raising predict response to fluid loading in critically ill patients. Chest 121:1245-1252

37. Haller M, Zollner C, Briegel J, Forst H (1995) Evaluation of a new continuous thermodilution cardiac output monitor in critically ill patients: a prospective criterion standard study. Crit Care Med 23:860-866

38. Ridel C, Lamia B, Monnet X, et al (2006) Passive leg raising and fluid responsiveness during spontaneous breathing: pulse contour evaluation. Intensive Care Med 32:S81 (abst)

39. Pinsky MR, Teboul JL (2005) Assessment of indices of preload and volume responsiveness. Curr Opin Crit Care 11:235-239

40. Vincent JL, Weil MH (2006) Fluid challenge revisited. Crit Care Med 34:1333-1337 